\title{
SOUND GAUSSIAN NOISE AND ITS CERTAIN TRANSFORMATION CAUSED BY MOVING BOUNDARY CONDITION EFFECT
}

\author{
R. Dyba and B. Zoltogorski
}

Institute of Telecommunication and Acoustics - Wroclaw Iechnical University - 50-37o Wroclow, Poland

Résumé.- Le but de cet article est de prëparer et de présenter une discussion de certains résultats sur la thêorie statistique des ondes acoustiques concernant une certaine transformation non linēaire du spectre du mouvement des sources d'onde. Du point de vue physique cette transformation non linéaire est due au caractère cinétique d'excitation des ondes. Ce mouvement de source à amplitude finie crée les déformations du spectre primaire, qui dépendent des deux causes suivantes : effet de condition de frontières mouvementées et effet de la propagation non linéaire. Notre attention est concentrée sur l'analyse de l'aspect mathëmatique du premier effet. Cela consiste à changer les variables de Lagrange en variables de Euler (L.E conversion) en admettant que le mouvement des sources d'onde est décrit par le processus stationnaire de Gauss. Cette méthode d'analyse est en partie basée sur les travaux de Rudenko et de Tchérkine, $/ 6,7 \%$, mais le résultat final des calculs théoriques est beaucoup plus complexe que Rudenko et Tchérkine le présentent, même si les équations de base dans les deux cas - la leur et la notre - du point de vue formel sont semblables. Il est nécessaire d'ajouter, que nous décrivons un autre phénomène physique. Nous avons déjà étudié ce problème auparavant mais par des méthodes approximatives, $/ 2 \%$

Abstract. - The purpose of the paper is to present and discuss a certain result of statistical wave theory which consists in determining a noni inear spectrum transformation caused by the kinematic character of the wave excitation. Large-amplitude source motion results in excitation spectrum distortions which are caused by two reasons : the moving boundary condition effect and the medium nonlinearity effect ; here attention is focussed on the first one. The essence of the paper consists in analysis of mathematical aspects of the exchange of the Lagrangian description to the Eulerian form (the so-called L-E conversion), when the source motion is to be Gaussian. The method presented here is partially based upon papers of Rudenko and Chirkin, $/ 6,7 /$, but the final result of theoretical calculations is more complex. It should be noted that a similar problem as considered here, was researched earlier but using approximate methods, $/ 2 \%$.

\section{1-INTRODUCTION- An intensive acoustical signal}

spreading out in a medium is submitted to nontinear deformations (connected to nonlinearity of hydrodynamic equations). According to the way of wave exci tations in the medium and to the frequency range of a propagated signal, one may accept different models of the medium (e.g. : ideal medium, medium with rosses, disperssive medium, etc.), and analysis of nonlinear effects may be carried out in different coordinate systems (Lagrangian variables, Eulerian variables). In the case of the kinematic way of wave excitations, apart from nonlinear effects corresponding to nonl inear propagation, an additional effect arises, which is closely connected to the movement of the source surface (the so-called moving boundary condition effect), $/ 1,2$, $3,4,5 \%$. It can be shown that in the low frequency range this effect results mainly in deformations of acoustical signal, and description of the effect is implied by conversion of Lagrangian coordinates into Eulerian coordinates. Most frequently this conversion is described by approximate methods.

A more complex and advanced situation is in the case of stochastic excitations of finite-amplitude acoustic waves. The problem has been solved for plane, spherical and cylindrical waves in a perfect gas by a method based upon Taylorian approximations of solutions of hydrodynamic equations. $(1,2)$.

Below an accurate method of analysis of acoustic Gaussian noise deformations is presented. This method is partially based upon the works of Rudenko and Chirkin $/ 6,7 /$.

2 - THEORY - Let $u(x, t)$ denote the particle velocity, and let $\xi(t)$ be a function describing the source surface motion. Assuming the medium to be lossiess and taking into account one-dimensional right-running propagation, the dependence between the particle velocity and the source motion has the following form, (Earnshow's equations), see e.g./B/:

$$
\begin{aligned}
& u(x, t)=\xi(\theta), \\
& t-\theta=\frac{x-\xi(\theta)}{c_{0}+\frac{\gamma+1}{2} \dot{\xi}(\theta)},
\end{aligned}
$$


where $c_{0}$ is small-signal sound velocity and $\gamma$ is the ratio of specific heats.

The problem may be formulated as follows: determine an analytical dependence between the particle velocity power spectrum and the source velocity power spectrum, when the following assumptions are accepted :

(i) the source motion is described by the stationary Gaussian process with the mean equal to zero ;

(ii) the source velocity power spectrum is concentrated at the range of sufficiently low frequencies, (cf. /1, 2, 3/).

Assumption (ii) needs an extra commentary. Namely it can be shown that the postulate about concentration of the "input" spectrum at the low frequency range is equivalent, in a certain sense, to situation when the nonlinear propagation effect is neglected (i.e. When the medium is assumed to be linear). Then it is sufficient to restrict ourselves to a description of the conversion of the moving boundary condition (given by $\xi(t)$ ) into the boundary condition $u(0, t)$ and next to obtain the dependence between the input spectrum and the output spectrum (i.e. the spectrum of $u(0, t)$ ). Since under the above established assumptions

$u(x, t)=u\left(0, t-\frac{x}{c_{0}}\right)$, then the particle velocity power spectrum is not the function of the space variable $x$.

Hence the starting equations take the form :

$u(0, t)=\dot{\xi}(\theta)$

$\theta=t+c_{0}^{-1} \xi(\theta)$.

Taking into account the Dirac delta function filtering property, $u(0, t)$ can be rewritten in such a manner :

$u(0, t)=\frac{1}{2 \pi} \int_{-\infty}^{\infty} \int_{-\infty}^{\infty} u(0, s) e^{i \omega(s-t)} d \omega d s$. (3)

Using eqs. (2a), (2b), (3) one obtains the integral dependence between $\xi(t)$ and $u(0, t)$, namely:

$$
\begin{aligned}
& u(0, t)=\frac{1}{2 \pi} \iint_{\infty}^{\infty} \int_{\infty}^{\infty}\left[\dot{\xi}(\theta)-c_{0}^{-1} \dot{\xi}^{2}(\theta)\right] \\
& \exp \left[-i \omega\left(\theta-c^{-1} \xi(\theta)-t\right)\right] d \theta d \omega ;
\end{aligned}
$$

by the way, it is worth noticing, that as opposed to eq. (2), the lest equation has a direct form.

Multiplying $u\left(0, t_{1}\right)$ by $u\left(0, t_{2}\right)$, which are given by eq. (4), and next averaging stochastically, we obtain the autocorrelation of the particle velocity in the form :

$$
\begin{aligned}
B_{u}\left(t_{1}, t_{2}, 0\right)=\frac{1}{4 \pi^{2}} \int_{-\infty}^{\infty} \int_{-\infty}^{\infty} e^{i\left(\omega_{1} t_{1}+\omega_{2} t_{2}\right)} \\
\int_{-\infty}^{\infty} \int_{-\infty}^{\infty} e^{-i\left(\omega_{1} \theta_{1}+\omega_{2} \theta_{2}\right)} \tilde{F} d \theta_{1} d \theta_{2} d \omega_{1} d \omega_{2},
\end{aligned}
$$

where

$$
\begin{aligned}
\tilde{F}= & \hat{F}\left(\theta_{1}, \theta_{7}, \omega_{1}, \omega_{2}\right) \\
= & E\left\{\left[\dot{\xi}\left(\theta_{1}\right)-c_{0}^{-1} \dot{\xi}^{2}\left(\theta_{1}\right)\right] \exp \left[i \frac{\omega_{1}}{0} \xi\left(\theta_{1}\right)\right]\right. \\
& {\left.\left[\dot{\xi}\left(\theta_{2}\right)-c_{0}^{-1} \dot{\xi}^{2}\left(\theta_{2}\right)\right] \exp \left[i \frac{\omega_{2}}{c_{0}} \xi\left(\theta_{2}\right)\right]\right\} }
\end{aligned}
$$

in which $E\{\cdot\}$ denotes the expected value operator. Performing substitutions under the integral twice over, and after it utilising the fact that $\tilde{F}$ is stationary with respect to the time axis translation, (i.e. $\tilde{F}\left(\theta_{1}, \theta_{2}, \omega_{1}, \omega_{2}\right)$ $\left.=F\left(\theta_{1}-\theta_{2}, \omega_{1}, \omega_{2}\right)=F\left(\theta, \omega_{1}, \omega_{2}\right)\right)$, we obtain: $B_{u}(\tau, 0)=\frac{1}{2 \pi} \int_{\infty}^{\infty} e^{i \omega \tau} \int_{\infty}^{\infty} e^{-i \omega \theta} F(\theta, \omega,-\omega) d \theta d \omega,(7)$ where $\tau=t_{1}-t_{2}$. 
By virtue of the Wiener-Khinchin theorem, the particle velocity power spectrum takes the form :

$S_{u}(\omega, 0)=S_{u}(\omega, x)=\int_{-\infty}^{\infty} e^{-i \omega \theta} F(\theta, \omega,-\omega) d \theta$

This equation has a direct form, but in consideration of the complicated dependence between $F$ and the parameters of the input process (i.e. $\xi(t)$ ), it is not convenient to further calculations and applications. Then we will pay some attention to transforming eq. (8) to a slightly simpler form. It can be shown that

$\tilde{F}\left(\theta_{1}, \theta_{2}, \omega,-\omega\right)=\frac{c_{0}^{2}}{\omega^{2}} \frac{\partial^{2}}{\partial \theta_{1} \partial \theta_{2}} A\left(\theta_{1}, \theta_{2}\right)$

$+\frac{16 c_{0}^{2}}{\omega^{4}} \frac{\partial^{4}}{\partial v_{1} \cdots \partial v_{4}} B\left(\nu_{1}, \ldots, v_{4}\right) \mid \begin{aligned} & v_{1}=v_{2}=\theta_{1} \\ & v_{3}=v_{4}=\theta_{2}\end{aligned}$

$-\left.\frac{4 c_{0}^{2}}{i \omega^{3}} \frac{\partial^{3}}{\partial v_{1} \partial v_{2} \partial v_{3}} c\left(v_{1}, v_{2}, v_{3}\right)\right|_{\begin{array}{l}v_{1}=v_{2}=\theta_{1} \\ v_{3}=\theta_{2}\end{array}}$

$+\left.\frac{4 c_{0}^{2}}{i \omega^{3}} \frac{\partial^{3}}{\partial \nu_{1} \partial v_{2} \partial v_{3}} D\left(\nu_{1}, v_{2}, v_{3}\right)\right|_{\begin{array}{l}v_{1}=\theta_{1} \\ v_{2}=v_{3}=\theta_{2}\end{array}}$

in which

$$
\begin{aligned}
& A\left(\theta_{1}, \theta_{2}\right)=C_{2}\left(\frac{\omega}{c_{0}},-\frac{\omega}{c_{0}}, \theta_{1}, \theta_{2}\right) \\
& \quad=\exp \left\{-\sigma^{2} \frac{\omega^{2}}{c_{0}^{2}}\left[1-R\left(\theta_{1}-\theta_{2}\right)\right]\right\},
\end{aligned}
$$

$B\left(\nu_{1}, \ldots, \nu_{4}\right)=c_{4}\left(\frac{\omega}{2 c_{0}}, \frac{\omega}{2 c_{0}},-\frac{\omega}{2 c_{0}},-\frac{\omega}{2 c_{0}}, \nu_{1}, \ldots, \nu_{4}\right)$

$$
=\exp \left\{-\sigma^{2}\left(\frac{\omega}{2 c_{0}}\right)^{2}\left[2+R\left(\nu_{1}-\nu_{2}\right)-R\left(\nu_{1}-\nu_{3}\right)\right.\right.
$$$$
-R\left(v_{1}-v_{4}\right)-R\left(v_{2}-v_{3}\right)-R\left(v_{2}-v_{4}\right)
$$$$
\left.\left.+R\left(v_{3}-v_{4}\right)\right]\right\}
$$

$c\left(v_{1}, v_{2}, v_{3}\right)=c_{3}\left(\frac{\omega}{2 c_{0}}, \frac{\omega}{2 c_{0}},-\frac{\omega}{c_{0}}, v_{1}, v_{2}, v_{3}\right)$

$$
\begin{aligned}
& =\exp \left\{-\sigma^{2} \frac{1}{2}\left(\frac{\omega}{c_{0}}\right)^{2}\left[\frac{3}{2}+\frac{1}{2} R\left(v_{1}-v_{2}\right)-R\left(y_{1}-v_{3}\right)\right.\right. \\
& \left.\left.+R\left(v_{2}-v_{3}\right)\right]\right\}
\end{aligned}
$$

$D\left(v_{1}, v_{2}, v_{3}\right)=c_{3}\left(\frac{\omega}{c_{0}},-\frac{\omega}{2 c_{0}}, \frac{\omega}{2 c_{0}}, v_{1}, v_{2}, v_{3}\right)$

$$
\begin{aligned}
& =\exp \left\{-\sigma^{2} \frac{1}{2}\left(\frac{\omega}{c_{0}}\right)^{2}\left[\frac{3}{2}-R\left(\nu_{1}-\nu_{2}\right)-R\left(\nu_{1}-\nu_{3}\right)\right.\right. \\
& \left.\left.+\frac{1}{2} R\left(\nu_{2}-\nu_{3}\right)\right]\right\}
\end{aligned}
$$

Where $c_{n}, n=2,3, n$, denotes the characteristic function of the $n$-dimentional probability density distribution of the raussian type and $R($.$) is the$ normalized autocorrelation of $\xi(t)$. Inserting eq. (9) into eq. (8), we obtain :

$S_{u}(\omega, 0)=\int_{-\infty}^{\infty} e^{-i \omega \theta} L\{P(\theta)\} \exp \left\{-\left(\frac{\sigma \omega}{c_{0}}\right)^{2}[1-R(\theta)]\right\} d \theta$

and next expandina the function $\exp \left[\left(\frac{\sigma \omega}{c_{0}}\right)^{2} R(\theta)\right]$ in the power series, after simple transformations we obtain the final formula for the narticle velocity power spectrum :

$s_{u}(\omega, 0)=\exp \left[-\left(\frac{\sigma \omega}{c_{0}}\right)^{2}\right] \sum_{n=0}^{\infty}\left(\frac{\sigma \omega}{c_{0}}\right)^{2 n} \frac{1}{n !} \int_{-\infty}^{\infty} e^{-j \omega \theta}$

$$
L\{R(\theta)\} R^{n}(\theta) d \theta
$$

where the differential operator $L\{R\}$ has the form: $L=-\sigma^{2}\left[\ddot{R}+\left(\frac{\sigma \omega}{c_{0}}\right)^{2} \dot{R}^{2}\right]+\frac{\sigma^{4}}{c_{0}^{2}}\left[2 \ddot{R}^{2}+4\left(\frac{\sigma \omega}{c_{0}}\right)^{2} \ddot{R} \ddot{p}^{2}\right.$ $\left.+2\left(\frac{\sigma \omega}{c_{0}}\right)^{2} \zeta R^{2}+\zeta^{2}+\left(\frac{\sigma \omega}{c_{0}^{4}}\right)_{R}^{4}\right\rceil+\frac{2 \cdot \sigma^{4} \omega}{i c_{0}^{2}}$ $\left[\begin{array}{cc}2 \ddot{R} \dot{R}+\zeta \dot{R}+\left(\frac{\sigma \omega}{c_{0}}\right)^{2} & \dot{R}^{3} \\ . . & . .\end{array}\right]$

in which $\dot{R}=\dot{R}(\theta), \ddot{R}=\ddot{R}(\theta)$ and $\zeta=\ddot{R}$ (0) 
3-. CONCLUSIONS

(i) Formula (4) is valid for any stochastic process describing the motion of the plane wave source ;

(ii) Formula (12) establishes the acurate solution of the problem of the L-E conversion when the source motion is of the Gaussian type.

The procedure outlined above may be used for low-frequency analysis of the finite-amplitude distorsion.

Finally, we would like to note that deterministic version of the said method was worked out by Kessler, /4/.

4-. REFERENCES

11 Dyba R., Contr. Papers of the 9th I.C.A. Madrid 1977, Vol. II, N-38, 746.

/2/ Dyba R. and Zoltogorski B., Spectral analysis of finite-amplitude effects for sound waves in ideal medium, Scientific Papers of the Institute of Telecommunication and Acoustics of Wroclaw Technical University, Monographs, Wroclaw 1978

13/ Frost P.A. and Harper E.Y., J. Acoust. Soc. Am., 1975, 58, 318

14/ Kessler H.C., J. Acoust. Soc. Am. 1962, 34 1958

15/ Zoltogorski B., Contr. Papers of the 9th ICA Madrid 1977, Vol II, N-35, 743

16/ Rudenko 0.V. and Chirkin A.S., Dok1 AN USSR, $1974,214,1045$.

17/ Rudenko 0.V. and Chirkin A.S., Dok1, AN USSR $1975,225,540$.

18/ BTackstock D.T., J. Acoust. Soc. Am., 1962, 34,9 